PROCEEDINGS OF THE

AMERICAN MATHEMATICAL SOCIETY

Volume 128, Number 11, Pages 3191-3200

S $0002-9939(00) 05484-8$

Article electronically published on May 11, 2000

\title{
ON THE NUMBER OF ABELIAN LEFT SYMMETRIC ALGEBRAS
}

\author{
KAREL DEKIMPE AND VEERLE ONGENAE
}

(Communicated by Christopher Croke)

\begin{abstract}
In this paper we prove that there are infinitely many abelian left symmetric algebras in dimensions $\geq 6$. Equivalently this means that there are, up to affine conjugation, infinitely many simply transitive affine actions of $\mathbb{R}^{k}$, for $k \geq 6$. This is a result which is usually credited to A.T. Vasquez, but for which there is no proof in the literature.
\end{abstract}

\section{INTRODUCTION}

Since a few years, there has been a growing interest in the study of simply transitive affine actions of Lie groups on some space $\mathbb{R}^{n}$. This interest is mostly due to the examples of Benoist ([2]) and Burde \& Grunewald ([3], [4]), who constructed connected and simply connected nilpotent Lie groups not admitting any simply transitive affine action on a space $\mathbb{R}^{n}$. These examples provided a negative answer to the question of Milnor ([12]) who asked whether any simply connected, connected solvable Lie group admits such an action. The study of such simply transitive affine actions is closely related (in fact equivalent) to the study of complete left-invariant affine structures on Lie groups, where an affine structure is a torsion free affine connection $([8],[7])$.

From another point of view, there is also the question of classifying (up to affine conjugation) all simply transitive and affine actions of a given Lie group $G$. This question, even in the case where $G=\mathbb{R}^{k}$ is abelian, seems to be very hard. Many authors refer to a thesis of H. Braverman (City University of New York, 1973), where all simply transitive affine actions of $\mathbb{R}^{2}$ and $\mathbb{R}^{3}$ are classified. In particular Braverman found that for $n=2$ there are 2 conjugacy classes, while for $n=3$ there are 5 conjugacy classes. One also finds many references to a result of A.T. Vasquez (e.g. 1], 10]) claiming to have proved that for $n=4$ and $n=5$ there are only finitely many conjugacy classes (without knowing the exact number), while for $n \geq 6$ there are infinitely many. However, there is no track in literature of a proof of this result.

In [10, H. Kim studied the simply transitive actions of all nilpotent Lie groups in dimensions $\leq 4$. In fact, $\mathrm{H}$. Kim works with the equivalent notion of complete

Received by the editors January 11, 1999.

2000 Mathematics Subject Classification. Primary 17A30, 17B30; Secondary 57M60, 53B05.

Key words and phrases. Left symmetric algebra, simply transitive affine action.

The first author is a Research Fellow of the Fund for Scientific Research - Flanders (Belgium) (F.W.O.). 
left symmetric algebras (see below). As a continuation of the work of Kim, we obtained in [5] the classification of all 5-dimensional, commutative, complete left symmetric algebras and therefore, we found, up to affine conjugation, all simply transive affine actions of $\mathbb{R}^{5}$. As a conclusion of this work we can count the number of conjugacy classes of simply transitive actions of $\mathbb{R}^{n}$ for $n \leq 5$. The result is given in the following table:

\begin{tabular}{r||c|c|c|c|c} 
Dimension & 1 & 2 & 3 & 4 & 5 \\
\hline Number of simply transitive affine actions & 1 & 2 & 5 & 12 & 37
\end{tabular}

This table shows that the results of Braverman and of Vasquez for $n \leq 5$ are correct.

In this paper we will establish a proof for the rest of Vasquez' result by giving explicitly an infinite family of conjugacy classes of simply transitive affine actions of $\mathbb{R}^{n}$ for $n \geq 6$.

\section{Simply transitive AFFine aCtions AND COMPLETE LEFT SYMMETRIC ALGEBRAS}

In this section we quickly recall how a simply transitive affine action of a Lie group is related to the notion of complete left symmetric structures. For more details and proofs, we refer the reader to [10], 8] and [6]. For simplicity, we restrict ourselves to the case of nilpotent Lie groups.

As usual we consider the group of affine motions of $\mathbb{R}^{n}$, denoted by $\operatorname{Aff}\left(\mathbb{R}^{n}\right)$ as being embedded into $\mathrm{Gl}_{n+1}(\mathbb{R})$. An affine map $\alpha: \mathbb{R}^{n} \rightarrow \mathbb{R}^{n}: x \mapsto A x+a$, with linear part $A \in \mathrm{Gl}_{n}(\mathbb{R})$ and translational part $a \in \mathbb{R}^{n}$ is thus identified with the element $\left(\begin{array}{cc}A & a \\ 0 & 1\end{array}\right)$. The Lie algebra $\mathfrak{a} f f\left(\mathbb{R}^{n}\right)$ of the Lie group $\operatorname{Aff}\left(\mathbb{R}^{n}\right)$ can therefore be viewed as the subalgebra of $\mathfrak{g} l_{n+1}(\mathbb{R})$ consisting of all matrices of the form $\left(\begin{array}{cc}A & a \\ 0 & 0\end{array}\right)$.

Let $N$ be any simply connected, connected nilpotent Lie group. An affine action of $N$ on a space $\mathbb{R}^{n}$ is given by a representation

$$
\varphi: N \rightarrow \operatorname{Aff}\left(\mathbb{R}^{n}\right): n \mapsto\left(\begin{array}{cc}
\varphi_{l}(n) & \varphi_{t}(n) \\
0 & 1
\end{array}\right)
$$

$\varphi_{l}(n)$ (resp. $\left.\varphi_{t}(n)\right)$ is called the linear (resp. translational) part of $\varphi(n)$. Moreover, such an action is simply transitive if and only if the map

$$
\varphi_{t}: N \rightarrow \mathbb{R}^{n}: n \mapsto \varphi_{t}(n)
$$

is bijective. In case $\varphi$ determines a simply transitive action, one knows that $\varphi(N)$ consists of unipotent matrices.

By taking the differential of the map $\varphi$, one obtains a Lie algebra morphism ( $\mathfrak{n}$ is the Lie algebra of $N)$ :

$$
\psi=d \varphi: \mathfrak{n} \rightarrow \mathfrak{a} f f\left(\mathbb{R}^{n}\right): X \mapsto\left(\begin{array}{cc}
\psi_{l}(X) & \psi_{t}(X) \\
0 & 0
\end{array}\right) .
$$

One has shown that $\varphi$ is a simply transitive action if and only if $\psi(\mathfrak{n})$ consists of nilpotent matrices and $\psi_{t}: \mathfrak{n} \rightarrow \mathbb{R}^{n}$ is bijective. Such a representation $\psi$ has been called an affine structure for $\mathfrak{n}$. 
Using an affine structure $\psi: \mathfrak{n} \rightarrow \mathfrak{a} f f\left(\mathbb{R}^{n}\right)$ of a nilpotent Lie algebra (as above), we can define a new product $\cdot: \mathfrak{n} \times \mathfrak{n} \rightarrow \mathfrak{n}$, which is given as follows:

$$
\cdot: \mathfrak{n} \times \mathfrak{n} \rightarrow \mathfrak{n}:(X, Y) \mapsto X \cdot Y:=\psi_{t}^{-1}\left(\psi_{l}(X)\left(\psi_{t}(Y)\right)\right) .
$$

One can easily check that this product satisfies the following two criteria:

$$
\left\{\begin{array}{c}
\forall X, Y, Z \in \mathfrak{n}:[X, Y] \cdot Z=X \cdot(Y \cdot Z)-Y \cdot(X \cdot Z), \\
\forall X, Y \in \mathfrak{n}:[X, Y]=X \cdot Y-Y \cdot X .
\end{array}\right.
$$

Definition 2.1. A vector space $V$ equipped with a bilinear product satisfying

$$
\forall X, Y, Z \in V:(X \cdot Y) \cdot Z-(Y \cdot X) \cdot Z=X \cdot(Y \cdot Z)-Y \cdot(X \cdot Z)
$$

is called a left symmetric algebra.

The two conditions above show that the product defined on $\mathfrak{n}$ turns it into a left symmetric algebra. Conversely, every left symmetric algebra $V$ with product · can also be viewed as a Lie algebra, by defining $[X, Y]=X \cdot Y-Y \cdot X, \forall X, Y \in V$. This Lie algebra is said to be associated to the left symmetric algebra.

Let $V$ be a left symmetric algebra; then one often denotes the left multiplication with an element $X$ by $\lambda(X)$, i.e. $\lambda(X) Y=X \cdot Y, \forall X, Y \in V$. Analogously, right multiplication is denoted by $\rho(X)$.

Definition 2.2. Let $V$ be a finite dimensional left symmetric algebra. This algebra is said to be complete if and only if the map $1+\rho(X): V \rightarrow V: Y \mapsto Y+\rho(X) Y$ is bijective for any $X \in V$.

It has been shown by Segal [13] (see also [9]) that completeness is equivalent to $\rho(X)$ being nilpotent for all $X$. Moreover, in case the associated Lie algebra is nilpotent, completeness is also equivalent to $\lambda(X)$ being nilpotent for any $X$.

Note that for the left symmetric algebra arising from an affine structure $\psi: \mathfrak{n} \rightarrow$ $\mathfrak{a} f f\left(\mathbb{R}^{n}\right)$, we have that

$$
\forall X \in \mathfrak{n}: \lambda(X)=\psi_{t}^{-1} \circ \psi_{l}(X) \circ \psi_{t}
$$

is nilpotent, since we already knew that $\psi_{l}(X)$ is nilpotent. It follows that the left symmetric structure arising from an affine structure is complete.

Conversely, let $V$, be a (finite dimensional) complete left symmetric algebra with a nilpotent associated Lie algebra structure. We can identify $V$ with $\mathbb{R}^{n}$ by choosing a basis $\left\{v_{1}, v_{2}, \ldots, v_{n}\right\}$ in $V$. If we use $\psi_{l}(X) \in \mathrm{Gl}_{n}(\mathbb{R})$ to denote the matrix, w.r.t. the chosen basis, representing the left multiplication map $\lambda(X), \forall X \in V$ and we use $\psi_{t}(X)$ to denote the coordinate of the element $X$, we find that the map

$$
\psi: V \rightarrow \mathfrak{a} f f\left(\mathbb{R}^{n}\right): X \mapsto\left(\begin{array}{cc}
\psi_{l}(X) & \psi_{t}(X) \\
0 & 0
\end{array}\right)
$$

determines an affine structure on the nilpotent Lie algebra $V$.

It is important to note that when we specialise to the case of associative (and hence left symmetric) algebras, the map $\psi$ constructed above is a faithful representation of algebras. Let us call this representation the natural representation of the algebra $V, \cdot$. (For general non-associative left symmetric algebras, this is of course never a representation). The following lemma will play a key role in our treatment of Vasquez' problem.

Lemma 2.3. Let $V_{1}$, and $V_{2}$, be two complete left symmetric algebras, which are even associative. Then $V_{1}, \cdot$ is isomorphic to $V_{2}, \cdot$ if and only if their natural representations $\psi_{1}\left(V_{1}\right)$ and $\psi_{2}\left(V_{2}\right)$ are affinely conjugate. 
Proof. Suppose that $\psi_{1}\left(V_{1}\right)$ and $\psi_{2}\left(V_{2}\right)$ are affinely conjugate. This implies that there exists an invertible matrix $\tilde{A}=\left(\begin{array}{cc}A & a \\ 0 & 1\end{array}\right) \in \operatorname{Aff}\left(\mathbb{R}^{n}\right)$ such that $\psi_{1}\left(V_{1}\right)=$ $\tilde{A} \psi_{2}\left(V_{2}\right) \tilde{A}^{-1}$. However, this implies that $\psi_{1}\left(V_{1}\right)$ and $\psi_{2}\left(V_{2}\right)$ are two matrix algebras which are conjugate to each other by means of an invertible matrix. This immediately implies that $\psi_{1}\left(V_{1}\right)$ and $\psi_{2}\left(V_{2}\right)$ are isomorphic algebra, and hence also $V_{1}$ and $V_{2}$ are isomorphic algebras.

We leave the other direction to the reader.

Another crucial observation is the fact that any commutative LSA is also associative. This follows easily from the definition of a LSA and the commutativity of the product:

$$
0=[X, Y] \cdot Z=X \cdot(Y \cdot Z)-Y \cdot(X \cdot Z) \Rightarrow 0=X \cdot(Z \cdot Y)-(X \cdot Z) \cdot Y
$$

showing the associativity of the product.

As a conclusion of all the above we can say that for a given abelian Lie algebra $\mathfrak{a}$, there is a correspondence between the complete left symmetric algebras, whose associated Lie algebra is $\mathfrak{a}$, and the affine structures on the Lie algebra $\mathfrak{a}$. Moreover, affinely equivalent affine structures (i.e. conjugate to each other with an element of $\operatorname{Aff}\left(\mathbb{R}^{n}\right)$ ) correspond to isomorphic left symmetric algebras and vice versa. It follows that counting the conjugacy classes of simply transitive affine actions of the abelian Lie group $\mathbb{R}^{n}$ is exactly the same as counting the isomorphism classes of commutative complete left symmetric algebras (i.e. those left symmetric algebras $\mathcal{L}$, with $X \cdot Y=Y \cdot X$, for all $X, Y \in \mathcal{L}$ ). (See also [11].)

In what follows we need a few more notions:

Definition 2.4. Let $\mathcal{L}$ be a left symmetric algebra. The center of $\mathcal{L}$, denoted by $C(\mathcal{L})$, is given as

$$
C(\mathcal{L})=\{X \in \mathcal{L} \mid X \cdot Y=Y \cdot X=0, \forall Y \in \mathcal{L}\}
$$

The trivial $n$-dimensional left symmetric algebra consists of the vector space $\mathbb{R}^{n}$ equipped with the zero product $\left(X \cdot Y=0, \forall X, Y \in \mathbb{R}^{n}\right)$. In case no ambiguity can arise, we also use $\mathbb{R}^{n}$ to denote this trivial left symmetric algebra.

It is obvious that the center of a left symmetric algebra is itself a trivial left symmetric algebra. It is also known that a complete, commutative left symmetric algebra always has a non-trivial center (e.g. see [5], [10]).

\section{INFINITELY MANY COMMUTATIVE LSAS}

In this section we construct in every dimension $\geq 6$ a family of commutative LSAs containing infinitely many non-isomorphic members. We start by giving an example of such a family in dimension six, which we thereafter generalize to every dimension $>6$.

Any $n$-dimensional commutative LSA $\mathcal{L}$ can be seen as an extension,

$$
0 \rightarrow \mathbb{R}^{k} \rightarrow \mathcal{L} \rightarrow \mathcal{K} \rightarrow 0,
$$

where $\mathbb{R}^{k}=C(\mathcal{L})$ is the center of $\mathcal{L}$ and $\mathcal{K}$ is an $(n-k)$-dimensional commutative LSA. In [5] we noticed that in dimension five the classification problem of the complete left symmetric commutative LSAs was quite complicated (and gave 
rise to more than a third of the five-dimensional commutative complete LSAs) for extensions of the form

$$
0 \rightarrow \mathbb{R}^{2} \rightarrow \mathcal{L} \rightarrow \mathbb{R}^{3} \rightarrow 0
$$

while all other cases of extensions of the form $0 \rightarrow \mathbb{R}^{k} \rightarrow \mathcal{L} \rightarrow \mathbb{R}^{5-k} \rightarrow 0$ were quite easy to handle.

Therefore, in dimension six it is obvious to search for an infinite family of commutative LSAs of the form

$$
0 \rightarrow \mathbb{R}^{2} \rightarrow \mathcal{L} \rightarrow \mathbb{R}^{4} \rightarrow 0 \quad \text { or } \quad 0 \rightarrow \mathbb{R}^{3} \rightarrow \mathcal{L} \rightarrow \mathbb{R}^{3} \rightarrow 0 .
$$

Our example will consist of extensions of the first kind, and are defined as follows:

Definition 3.1. For any parameter $\mu \in \mathbb{R}$, with $\mu>1$ we define a 6 -dimensional algebra $\mathcal{L}_{\mu}$ which is given as a vector space with basis $\left\{e_{1}, e_{2}, e_{3}, e_{4}, e_{5}, e_{6}\right\}$ and where the bilinear product is given by

$e_{1} e_{1}=e_{5}, e_{2} e_{2}=e_{6}, e_{3} e_{3}=e_{5}+e_{6}, e_{4} e_{4}=e_{5}+\mu e_{6}, e_{i} e_{j}=0$ in all other cases.

Lemma 3.2. For any value of the parameter $\mu(\mu \in \mathbb{R}$ and $\mu>1)$, the algebra $\mathcal{L}_{\mu}$ is commutative, left symmetric and complete. The center of $\mathcal{L}$ is two-dimensional and has $\left\{e_{5}, e_{6}\right\}$ as a basis.

Proof. It is obvious from the definition that the product in $\mathcal{L}_{\mu}$ is commutative. Moreover, for any $X, Y, Z \in \mathcal{L}_{\mu}$, we have that $X \cdot(Y \cdot Z)=(X \cdot Y) \cdot Z=0$, from which it trivially follows that this product is left symmetric (even associative). The assertion about the center is also easy to check.

Theorem 3.3. Let $\mu_{1}>1$ and $\mu_{2}>1$ be two real parameters. If $\mathcal{L}_{\mu_{1}}$ and $\mathcal{L}_{\mu_{2}}$ are isomorphic, then $\mu_{1}=\mu_{2}$.

Proof. Assume that $\mathcal{L}_{\mu_{1}}$ and $\mathcal{L}_{\mu_{2}}$ are isomorphic. To avoid confusion, we denote the basis of $\mathcal{L}_{\mu_{1}}$ by $\left\{e_{1}, e_{2}, \ldots, e_{6}\right\}$ (as in Definition 3.1) and that of $\mathcal{L}_{\mu_{2}}$ with $\left\{f_{1}, f_{2}, \ldots, f_{6}\right\}$. Let $\eta$ be an isomorphism from $\mathcal{L}_{\mu_{1}}$ to $\mathcal{L}_{\mu_{2}}$ and let $Y=\left(y_{i j}\right)_{1 \leq i, j \leq 6}$ be the matrix of $\eta$, with respect to the given bases. We use the convention that the $i$-th column indicates the image of the $i$-th basis vector, i.e. $\eta\left(e_{i}\right)=\sum_{j=1}^{6} y_{j i} f_{j}$.

As the isomorphism $\eta$ maps $C\left(\mathcal{L}_{\mu_{1}}\right)$ to $C\left(\mathcal{L}_{\mu_{2}}\right)$, the matrix $Y$ is of the form

$$
\begin{gathered}
\left(\begin{array}{llllll}
y_{11} & y_{12} & y_{13} & y_{14} & 0 & 0 \\
y_{21} & y_{22} & y_{23} & y_{24} & 0 & 0 \\
y_{31} & y_{32} & y_{33} & y_{34} & 0 & 0 \\
y_{41} & y_{42} & y_{43} & y_{44} & 0 & 0 \\
y_{51} & y_{52} & y_{53} & y_{54} & y_{55} & y_{56} \\
y_{61} & y_{62} & y_{63} & y_{64} & y_{65} & y_{66}
\end{array}\right), \text { i.e. } \\
\left\{\begin{array}{llll}
y_{15}= & 0 & (1) \\
y_{16} & = & 0 & (2) \\
y_{25}= & 0 & (3) \\
y_{26}= & 0 & (4) \\
y_{35}= & 0 & (5) \\
y_{36}= & 0 & (6) \\
y_{45}= & 0 & (7) \\
y_{46}= & 0 & (8)
\end{array}\right.
\end{gathered}
$$


Of course, we can write down more conditions on the entries of $Y$. E.g. from $\eta\left(e_{1} \cdot e_{1}\right)=\eta\left(e_{1}\right) \cdot \eta\left(e_{1}\right)$, it follows that

$$
\begin{aligned}
\eta\left(e_{5}\right) & =y_{55} f_{5}+y_{65} f_{6} \\
& =\left(y_{11} f_{1}+y_{21} f_{2}+\cdots+y_{61} f_{6}\right) \cdot\left(y_{11} f_{1}+y_{21} f_{2}+\cdots+y_{61} f_{6}\right) \\
& =y_{11}^{2} f_{5}+y_{21}^{2} f_{6}+y_{31}^{2}\left(f_{5}+f_{6}\right)+y_{41}^{2}\left(f_{5}+\mu_{2} f_{6}\right)
\end{aligned}
$$

which leads to the equations $y_{55}=y_{11}^{2}+y_{31}^{2}+y_{41}^{2}$ and $y_{65}=y_{21}^{2}+y_{31}^{2}+\mu_{2} y_{41}^{2}$.

Making the analogous computations $\eta\left(e_{i} \cdot e_{j}\right)=\eta\left(e_{i}\right) \cdot \eta\left(e_{j}\right)$, for all $1 \leq i \leq j \leq 6$, gives rise to the following set of conditions on the entries of $Y$.

$$
\left\{\begin{array}{l}
y_{55}=y_{11}^{2}+y_{31}^{2}+y_{41}^{2} \\
y_{56}=y_{12}^{2}+y_{32}^{2}+y_{42}^{2} \\
y_{65}=y_{21}^{2}+y_{31}^{2}+\mu_{2} y_{41}^{2} \\
y_{66}=y_{22}^{2}+y_{32}^{2}+\mu_{2} y_{42}^{2}
\end{array}\right.
$$

and

$$
\left\{\begin{array}{llll}
y_{11} y_{14}+y_{31} y_{34}+y_{41} y_{44}= & 0 & (13) \\
y_{12} y_{14}+y_{32} y_{34}+y_{42} y_{44}= & 0 & (14) \\
y_{13} y_{14}+y_{33} y_{34}+y_{43} y_{44} & = & 0 & (15) \\
y_{21} y_{24}+y_{31} y_{34}+\mu_{2} y_{41} y_{44}= & 0 & (16) \\
y_{22} y_{24}+y_{32} y_{34}+\mu_{2} y_{42} y_{44}= & 0 & (17) \\
y_{23} y_{24}+y_{33} y_{34}+\mu_{2} y_{43} y_{44}= & 0 & (18) \\
y_{11} y_{12}+y_{31} y_{32}+y_{41} y_{42}= & 0 & (19) \\
y_{11} y_{13}+y_{31} y_{33}+y_{41} y_{43}= & 0 & (20) \\
y_{12} y_{13}+y_{32} y_{33}+y_{42} y_{43} & = & 0 & (21) \\
y_{21} y_{22}+y_{31} y_{32}+\mu_{2} y_{41} y_{42}= & 0 & (22) \\
y_{21} y_{23}+y_{31} y_{33}+\mu_{2} y_{41} y_{43}= & 0 & (23) \\
y_{22} y_{23}+y_{32} y_{33}+\mu_{2} y_{42} y_{43}= & 0 & (24)
\end{array}\right.
$$

and

$$
\left\{\begin{array}{lll}
-y_{11}^{2}-y_{12}^{2}+y_{13}^{2}-y_{31}^{2}-y_{32}^{2}+y_{33}^{2}-y_{41}^{2}-y_{42}^{2}+y_{43}^{2} & = & 0 \\
-y_{21}^{2}-y_{22}^{2}+y_{23}^{2}-y_{31}^{2}-y_{32}^{2}+y_{33}^{2}-\mu_{2} y_{41}^{2}-\mu_{2} y_{42}^{2}+\mu_{2} y_{43}^{2} & = & 0 \\
-y_{11}^{2}-\mu_{1} y_{12}^{2}+y_{14}^{2}-y_{31}^{2}-\mu_{1} y_{32}^{2}+y_{34}^{2}-y_{41}^{2}-\mu_{1} y_{42}^{2}+y_{44}^{2} & = & 0 \\
-y_{21}^{2}-\mu_{1} y_{22}^{2}+y_{24}^{2}-y_{31}^{2}-\mu_{1} y_{32}^{2}+y_{34}^{2}-\mu_{2} y_{41}^{2}-\mu_{1} \mu_{2} y_{42}^{2}+\mu_{2} y_{44}^{2} & = & 0
\end{array}\right.
$$

Moreover as $\eta$ is an isomorphism, the determinant of $Y$, denoted by $|Y|$, cannot be zero, or by (1), (2), (3), (4), (5), (6), (7) and (8),

$$
|Y|=\left|\begin{array}{llll}
y_{11} & y_{12} & y_{13} & y_{14} \\
y_{21} & y_{22} & y_{23} & y_{24} \\
y_{31} & y_{32} & y_{33} & y_{34} \\
y_{41} & y_{42} & y_{43} & y_{44}
\end{array}\right|\left|\begin{array}{ll}
y_{55} & y_{56} \\
y_{65} & y_{66}
\end{array}\right| \neq 0
$$

Observe that the equations (13), (14) and (15) can be seen as a homogeneous system of equations in the variables $y_{14}, y_{34}$ and $y_{44}$ with coefficient matrix

$$
Y_{1}=\left(\begin{array}{lll}
y_{11} & y_{31} & y_{41} \\
y_{12} & y_{32} & y_{42} \\
y_{13} & y_{33} & y_{43}
\end{array}\right)
$$


If the determinant of $Y_{1}$ is not zero, then $\left(y_{14}, y_{34}, y_{44}\right)$ has to be $(0,0,0)$. By equation (27) and because $\mu_{1}>1$, we have that

$$
y_{11}=y_{12}=y_{31}=y_{32}=y_{41}=y_{42}=0 \text {. }
$$

But this implies that the determinant of $Y$ would be zero:

$$
|Y|=\left|\begin{array}{cccc}
0 & 0 & y_{13} & 0 \\
y_{21} & y_{22} & y_{23} & y_{24} \\
0 & 0 & y_{33} & 0 \\
0 & 0 & y_{43} & 0
\end{array}\right|\left|\begin{array}{cc}
0 & 0 \\
y_{21}^{2} & y_{22}^{2}
\end{array}\right|=0
$$

Consequently the determinant of $Y_{1}$ is zero.

Now we regard the equations (16), (17) and (18) as a homogeneous system of equations in the variables $y_{24}, y_{34}$ and $y_{44}$. In an analogous way, using equation (28), it can be shown that the determinant of this system of equation has to be zero. Hence,

$$
\left|\begin{array}{lll}
y_{11} & y_{31} & y_{41} \\
y_{12} & y_{32} & y_{42} \\
y_{13} & y_{33} & y_{43}
\end{array}\right|=0
$$

and

$$
\left|\begin{array}{lll}
y_{21} & y_{31} & \mu_{2} y_{41} \\
y_{22} & y_{32} & \mu_{2} y_{42} \\
y_{23} & y_{33} & \mu_{2} y_{43}
\end{array}\right|=\mu_{2}\left|\begin{array}{lll}
y_{21} & y_{31} & y_{41} \\
y_{22} & y_{32} & y_{42} \\
y_{23} & y_{33} & y_{43}
\end{array}\right|=0 .
$$

Let $v_{1}, v_{2}, v_{3}, v_{4}, v_{5}$ and $v_{6}$ be the following vectors of 3-dimensional Euclidean space $\mathbb{R}^{3}$.

$$
\begin{aligned}
& v_{1}=\left(\begin{array}{l}
y_{11} \\
y_{31} \\
y_{41}
\end{array}\right), v_{2}=\left(\begin{array}{l}
y_{12} \\
y_{32} \\
y_{42}
\end{array}\right), v_{3}=\left(\begin{array}{l}
y_{13} \\
y_{33} \\
y_{43}
\end{array}\right), \\
& v_{4}=\left(\begin{array}{l}
y_{21} \\
y_{31} \\
y_{41}
\end{array}\right), v_{5}=\left(\begin{array}{l}
y_{22} \\
y_{32} \\
y_{42}
\end{array}\right), v_{6}=\left(\begin{array}{l}
y_{23} \\
y_{33} \\
y_{43}
\end{array}\right) .
\end{aligned}
$$

The equations (19), (20) and (21) express that

$$
v_{1} \perp v_{2}, v_{1} \perp v_{3}, v_{2} \perp v_{3} .
$$

Moreover by (3.29), $v_{1}, v_{2}$ and $v_{3}$ are linear dependent. This means that at least one of the vectors has to be the zero vector. The equation (25) can be interpreted as

$$
\left\|v_{3}\right\|^{2}=\left\|v_{1}\right\|^{2}+\left\|v_{2}\right\|^{2}
$$

If $v_{3}=0$, then $v_{1}=v_{2}=0$ and the determinant of $Y$ would be zero. Thus $v_{3} \neq 0$. This leaves two possible cases, namely $v_{1}=0$ or $v_{2}=0$ (but not both at the same time).

$$
\text { Case 1: } v_{1}=\left(y_{11}, y_{31}, y_{41}\right)=(0,0,0)
$$

As the determinant of $Y$ might not be zero, the entry $y_{21}$ is not zero and the vector $v_{4}$ is not the zero vector. By equations (16), (22) and (23), we have that

$$
y_{22}=y_{23}=y_{24}=0 \text {. }
$$


Consequently,

$$
v_{4} \perp v_{5} \text { and } v_{4} \perp v_{6} .
$$

By (3.30), $v_{4}, v_{5}$ and $v_{6}$ are linear dependent. Because $v_{4}$ is not the zero vector, $v_{5}$ and $v_{6}$ must be linear dependent. The equation (26) is now

$$
-y_{21}^{2}-y_{32}^{2}+y_{33}^{2}-\mu_{2} y_{42}^{2}+\mu_{2} y_{43}^{2}=0 \text {. }
$$

This shows that $v_{6}=\left(0, y_{33}, y_{43}\right)$ might not be the zero vector. Thus, as $v_{5}$ and $v_{6}$ are linear dependent, there exists a real number $\lambda$ such that

$$
y_{32}=\lambda y_{33} \text { and } y_{42}=\lambda y_{43} .
$$

The equation (24) is then

$$
\lambda\left(y_{33}^{2}+\mu_{2} y_{43}^{2}\right)=0 .
$$

As $v_{6}$ is not zero and $\mu_{2}>1, \lambda$ must be zero and $v_{5}$ is the zero vector, i.e. $y_{32}=y_{42}=0$. Because $v_{2} \neq 0, y_{12} \neq 0$. As a consequence, by the equations (14) and (21), $y_{13}=y_{14}=0$. The remaining conditions on the entries of $Y$ ((15), (18), $(25),(26),(27)$ and $(28))$ are now:

$$
\left\{\begin{array}{llll}
y_{33} y_{34}+y_{43} y_{44} & = & 0 & (31) \\
y_{33} y_{34}+\mu_{2} y_{43} y_{44} & = & 0 & (32) \\
-y_{12}^{2}+y_{33}^{2}+y_{43}^{2} & = & 0 & (33) \\
-y_{21}^{2}+y_{33}^{2}+\mu_{2} y_{43}^{2}= & 0 & (34) \\
-\mu_{1} y_{12}^{2}+y_{34}^{2}+y_{44}^{2}= & 0 & (35) \\
-y_{21}^{2}+y_{34}^{2}+\mu_{2} y_{44}^{2} & = & 0 & (36)
\end{array}\right.
$$

Because $\mu_{2}>1$, the equations (31) and (32) imply that

$$
y_{33} y_{34}=0 \text { and } y_{43} y_{44}=0 .
$$

Hence, as the determinant of $Y$ must not be zero, this leaves two possible cases,

$$
y_{33}=y_{44}=0 \text { or } y_{43}=y_{34}=0 .
$$

Note that in the first case $y_{43}$ and $y_{34}$ cannot be zero, and in the second case $y_{33}$ and $y_{44}$. Consequently,

$$
\left(y_{12}^{2}=y_{43}^{2}=\frac{y_{21}^{2}}{\mu_{2}}=\frac{y_{34}^{2}}{\mu_{1}}=\frac{y_{34}^{2}}{\mu_{2}}\right) \text { or }\left(y_{12}^{2}=y_{33}^{2}=y_{21}^{2}=\frac{y_{44}^{2}}{\mu_{1}}=\mu_{2} y_{44}^{2}\right) .
$$

Because $\mu_{1}>1$ and $\mu_{2}>1, \mu_{2}$ cannot be equal to $\frac{1}{\mu_{1}}$ and the second case is not possible. We conclude that $\mu_{1}=\mu_{2}$.

Case 2: $v_{2}=\left(y_{12}, y_{32}, y_{42}\right)=(0,0,0)$

This case can be treated in the same way, by changing $v_{1}$ and $v_{4}$, respectively, by $v_{2}$ and $v_{5}$ and vice versa. The conclusion is again that $\mu_{1}$ equals $\mu_{2}$.

In an analogous way this example can be generalized to every dimension $n \geq 6$.

Definition 3.4. Let $n \geq 6$ be any integer. For any parameter $\mu \in \mathbb{R}$, with $\mu>1$ we define a $n$-dimensional algebra $\mathcal{L}_{\mu}^{n}$ which is given as a vector space with basis $\left\{e_{1}, e_{2}, \ldots, e_{n}\right\}$ and where the bilinear product is given by

$e_{1} e_{1}=e_{5}, e_{2} e_{2}=e_{6}, e_{3} e_{3}=e_{5}+e_{6}, e_{4} e_{4}=e_{5}+\mu e_{6}, e_{i} e_{j}=0$ in all other cases. 
As in Lemma 3.2, one can show that any algebra $\mathcal{L}_{\mu}^{n}$ is a commutative, complete left symmetric algebra. We can now prove

Theorem 3.5. Let $n \geq 6$ be any integer and let $\mu_{1}>1$ and $\mu_{2}>1$ be two real parameters. If $\mathcal{L}_{\mu_{1}}^{n}$ and $\mathcal{L}_{\mu_{2}}^{n}$ are isomorphic, then $\mu_{1}=\mu_{2}$.

Proof. As in the proof of Theorem 3.3. we denote the basis elements of $\mathcal{L}_{\mu_{1}}^{n}$ by symbols $e_{i}(1 \leq i \leq n)$ and those of $\mathcal{L}_{\mu_{2}}^{n}$ by $f_{i}(1 \leq i \leq n)$. Suppose that $\eta: \mathcal{L}_{\mu_{1}}^{n} \rightarrow$ $\mathcal{L}_{\mu_{2}}^{n}$ is an isomorphism; then $\eta\left(C\left(\mathcal{L}_{\mu_{1}}^{n}\right)\right)=\eta\left(C\left(\mathcal{L}_{\mu_{2}}^{n}\right)\right)$ and $\eta\left(\mathcal{L}_{\mu_{1}}^{n} \cdot \mathcal{L}_{\mu_{1}}^{n}\right)=\mathcal{L}_{\mu_{2}}^{n} \cdot \mathcal{L}_{\mu_{2}}^{n}$. This last equality implies that $\eta\left\langle e_{5}, e_{6}\right\rangle=\left\langle f_{5}, f_{6}\right\rangle$. As $\left\langle e_{7}, e_{8}, \ldots, e_{n}\right\rangle \oplus\left\langle e_{5}, e_{6}\right\rangle=$ $C\left(\mathcal{L}_{\mu_{1}}^{n}\right)$, we must have that $\eta\left(\left\langle e_{7}, e_{8}, \ldots, e_{n}\right\rangle\right) \oplus \eta\left(\left\langle e_{5}, e_{6}\right\rangle\right)=C\left(\mathcal{L}_{\mu_{2}}^{n}\right)$. It follows that $\eta$ induces a LSA-isomorphism between

$$
\bar{\eta}: \mathcal{L}_{\mu_{1}}^{n} /\left\langle e_{7}, e_{8}, \ldots, e_{n}\right\rangle \rightarrow \mathcal{L}_{\mu_{2}}^{n} /\left(\eta\left(\left\langle e_{7}, e_{8}, \ldots, e_{n}\right\rangle\right)\right) .
$$

However, $\mathcal{L}_{\mu_{1}}^{n} /\left\langle e_{7}, e_{8}, \ldots, e_{n}\right\rangle \cong \mathcal{L}_{\mu_{1}}$ and $\mathcal{L}_{\mu_{2}}^{n} /\left(\eta\left(\left\langle e_{7}, e_{8}, \ldots, e_{n}\right\rangle\right)\right) \cong \mathcal{L}_{\mu_{2}}$, implying that (Theorem 3.3) $\mu_{1}=\mu_{2}$.

Corollary 3.6. Every $n$-dimensional $(n \geq 6)$ abelian Lie algebra has infinitely (uncountable) many non-isomorphic left symmetric structures.

As explained in the previous section, this leads to the "infinite" part of Vasquez' result.

Corollary 3.7. There are infinitely many conjugacy classes of abelian Lie subgroups of $\operatorname{Aff}\left(\mathbb{R}^{n}\right)$ acting simply transitively on $\mathbb{R}^{n}$ for any $n \geq 6$.

\section{REFERENCES}

1. Auslander, L. Simply Transitive Groups of Affine Motions. Amer. J. Math., 1977, 99 (4), pp. 809-826. MR 56:5782

2. Benoist, Y. Une nilvariété non affine. J. Differential Geom., 1995, 41 pp. 21-52. MR 96c:53077

3. Burde, D. Affine structures on nilmanifolds. Internat. J. Math, 1996, 7 5, pp. 599 - 616 . MR 97i:53056

4. Burde, D. and Grunewald, F. Modules for certain Lie algebras of maximal class. J. Pure Appl. Algebra, 1995, 99 pp. 239-254. MR 96d:17007

5. Dekimpe, K., Igodt, P., and Ongenae, V. The five-dimensional complete left symmetric algebra structures compatible with an abelian Lie algebra structure. Linear Algebra and its Applications, 1997, 263, pp. 349-375. MR 98g:17001

6. Dekimpe, K. and Malfait, W. Affine structures on a class of virtually nilpotent groups. Topol. and its Applications, 1996, 73 (2), pp. 97-119. MR 97j:57060

7. Fried, D., Goldman, W., and Hirsch, M. Affine manifolds with nilpotent holonomy. Comment. Math. Helv., 1981, 56 pp. 487-523.

8. Fried, D. and Goldman, W. M. Three-Dimensional Affine Crystallographic Groups. Adv. in Math., 1983, 47 1, pp. 1-49. MR 84d:20047

9. Helmstetter, J. Radical d'une algèbre symérique a gauche. Ann. Inst. Fourier Grenoble, 1979, 29 (4), pp. 17-35. MR 81j:17002

10. Kim, H. Complete left-invariant affine structures on nilpotent Lie groups. J. Differential Geom., 1986, 24, pp. 373-394. [MR 88c:53030

11. Matsushima, Y. Affine structures on complex manifolds. Osaka J. Math, 1968, 5, pp. 215-222. MR 39:2086 
12. Milnor, J. On fundamental groups of complete affinely flat manifolds. Adv. Math., 1977, 25 pp. 178-187. MR 56:13130

13. Segal, D. The structure of complete left-symmetric algebras. Math. Ann., 1992, 293 (3), pp. 569-578. MR 93i: 17026

Katholieke Universiteit Leuven Campus Kortrijk, Universitaire Campus, B-8500 KortriJK, BelgiUm

E-mail address: Karel.Dekimpe@kulak.ac.be

Katholieke Universiteit Leuven Campus Kortrijk, Universitaire Campus, B-8500 KortriJK, Belgium

Current address: Department of Pure Mathematics and Computer Algebra, University of Ghent, Galglaan 2, B-9000 Gent, Belgium

E-mail address: vo@cage.rug.ac.be 